\title{
Image conscious
}

\section{The main theme this week is image acquisition and handling.}

\section{Manual control}

Bio-Rad

www.bio-rad.com

\section{Stay in the picture}

A new manual control unit has been introduced to allow users of Radiance laser scanning microscopes to fine-tune and optimize acquisition settings without taking their eyes away from the image on the screen. Instead of using a mouse or keyboard to navigate between on-screen icons, users can manually adjust parameters such as PMT gain, laser power (attenuation) and confocal aperture (iris) via touch-sensitive dials and buttons. Instrument settings can be adjusted progressively with one hand, and the control layout is suitable for either left- or right-handed operation.

Reader Service No. 100

\section{BioDoc-lt}

UVP www.uvp.com

Compact image acquisition system

The BioDoc-It system combines a CCD video camera, integrated LCD monitor, touchpad control panel, disk drive, darkroom enclosure and 302-nm UV benchtop transilluminator. It is designed for gel documentation and archiving of fluorescent and non-fluorescent gels, membranes, blots, film, plates and assays. A live preview function allows researchers to acquire exactly what is shown on the monitor. Exposure times can be pre-set for integration of the camera from 0.1 up to 10 seconds, while an auto-save function saves up to four images on disk. An optional thermal printer and UVto-white-light converter are also available.

Reader Service No. 101

\section{NucleoVision 920}

NucleoTech www.nucleotech.com

\section{Versatile chemiluminescence workstation}

Based on GelExpert imaging software, this workstation supports applications involving chemiluminescence, fluorescence and visible light imaging and can be used to document and analyse blots, gels, autoradiographs and 3-D QPCR, dot blot and ELISA. The integrated software offers facilities for image preprocessing, annotation, enhancement, analysis, archiving and publication. Analytical tools include OD analysis, molecular weight calculation, 1-D automated multi-lane densitometry, Dot/Slot blot analysis, free object quantification, 2-D spot densitometry and dynamic image capture. The system supports images from CCD cameras, TWAINcompatible scanners and phosphor imagers. Reader Service No. 102

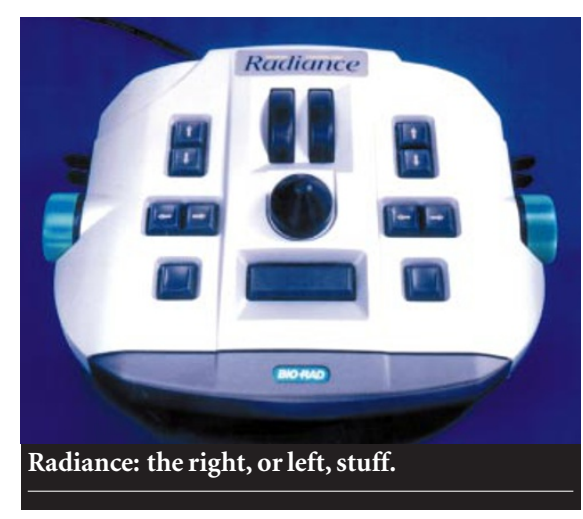

GP-KS1000

Panasonic www.panasonic.com/indvision This one will make you see red

The digital signal processing GP-KS1000 colour microcamera features a high-density $\mathrm{CCD}(830 \times 970 \mathrm{~V}$ pixel array $)$ that produces 560 lines of resolution with a signal-to-noise ratio of $54 \mathrm{~dB}$. The camera head measures $17 \mathrm{~mm}$ in diameter and $42 \mathrm{~mm}$ in length, without the lens. Its RGB colour filter reproduces a full spectrum of colours, including reds that would normally be expected using a three-CCD camera. The unit comes with an auto/manual electronic shutter, built-in fullfield colour bars, vertical and horizontal edge enhancement and four selectable user files for storage and retrieval of specific camera settings.

Reader Service No. 103

\section{MetaMorph 4.5}

Universal Imaging Corporation

www.image1.com

Streamlined interface with advanced features

Refinements to the MetaMorph bioimaging software program include a simplified user interface and more advanced features. Application-specific toolbars such as Fluorescence Tools can be used to acquire multiple wavelengths from digital cameras, measure colocalization between probes and overlay images. A new Acquire dialogue box sets camera binning, gain and auto-exposure, controls camera shutters during acquisition and defines exposure times and acquisition regions. Gray values in biological samples can be quantified using the Region Measurements function to combine measurements of intensity, area and other parameters. MetaMorph 4.5 comes with a training $\mathrm{CD}$ and runs under Windows 95/98 and NT. The program supports a variety of black-and-white and colourcooled CCD cameras from major manufacturers.

Reader Service No. 104
DXM 1200 digital camera

Nikon

www.nikon.com

Definitely worth looking into

Aimed at microscopists who need to take and store large numbers of high-quality digital photomicrographs, the DXM 12001.4 million CCD produces images of 12 million output pixels that can be transferred to a computer at speeds of up to 12 frames per second. Data can be saved in TIFF, JPEG or BMP format. Shooting conditions and live, still and thumbnail formats can be viewed on screen simultaneously, switching between live and still as required. Exposures can be made while monitoring high-quality live images, with the camera set to print each time an exposure is made for immediate verification of image capture. Reader Service No. 105

Dual-action manifold

Bio-Tek

www.biotek.com Quadruples the capacity of ELX50 washers

This 16-channel manifold offers the facility to switch between 96- and 384-well plates with no need to change washer hardware. Dispense and aspiration positions are controlled independently for overfill washing and overflow protection. Adjustable dispense and aspiration rates allow the washer to be used in a wide range of applications, including cell-based assays. An optional external valve allows automatic dispensing of up to four reagents. Up to 75 user-defined programs can be created and stored on-board, with a facility to run up to 10 programs together for complex wash routines. Reader Service No. 106

These notes are compiled in the Nature office from information provided by the manufacturers.

ADVERTISEMENT

Custom Peptides \& Polyclonal Antibodies

Please contact us about complete services for your custom peptide and polyclonal antibody needs.

\section{Research Genetics \\ U.S. or Canada 800-533-4363 FAX 256-536-9016}

Check out our homepage at http://www.resgen.com

READER ENQUIRY NO. 6 